\title{
CARDIAC TRANSPLANT -A SINGLE CENTRE RETROSPECTIVE OBSERVATION
}

Anaesthesiology

Parthasarathy

Laxmanan

\section{Kayalvizhi K}

Balasundaram*

Kalaivani Nadar

Vijayasankar

Muthu

\section{Charankumar Natarajan}

M.D, D.A., Professor And Hod, Dept Of Anaesthesiology, Tamilnadu Govt Multisuperspeciality Hospital Chennai.

M.D, Assistant Professor, Anaesthesiology, Tamilnadu Govt Multisuperspeciality Hospital Chennai. * Corresponding Author

M.D., Assistant Professor, Anaesthesiology, Tamilnadu Govt Multisuper Speciality Hospital, Chennai.

M.D., Associate Professor, Anaesthesiology Tamilnadu Govt Multisuperspeciality Hospital Chennai.

M.B.B.S, D.A., Assistant Surgeon, Nagapattinam Govt Hospital (Previously Pg Student In Omandurar Hospital).

\section{ABSTRACT}

Background And Aim:Advances in pharmacological and nonpharmacological management of heart failure shifted the paradigm to transplantation of heart. Currently so many centers are doing heart transplant as the availability of donors and recipients are increasing day by day. The goal of this study is to share our experience in all our heart transplantation procedures.

Ours is a tertiary care government multi super Speciality hospital. In our institute we have been doing cardiac surgeries for six years and heart transplants for past three years. In this discussion we share our experience about how we did all the procedures in our center.

Method: After getting approval from institutional research committee we analyzed 8 transplants done in our center. The preoperative optimization, monitoring tools, anesthetic technique and post-operative complications and management are discussed. Apart from routine monitors we have used BIS, Cerebral oximetry and cardiac output monitors.

Result:Of the eight cases, six are doing well including a (pediatric) 10-year-old recipient. Of the remaining two, one patient died on 3rd PostOperative Day due to acute kidney injury and the other was death due to acute rejection.

Conclusion:The key points we have learnt from our experience are careful selection and preparation of the donor, adequate preload with optimal inotropic support during weaning, minimizing increase in pulmonary vascular resistance and good pain relief are key aspects for successful outcome.

\section{KEYWORDS}

Cardiac Surgical Procedures, Heart Transplantation, Cardiac Output, Pulmonary Vascular Resistance.

\section{INTRODUCTION}

In India the first heart transplant was first performed in 1994 at All India Institute of Medical Sciences in India. The advancement in both pharmacological \& nonpharmacological management of heart failure and use of ventricular assist devices \& ECMO increases the number of survivors. Following this in recent past there is an increase in number of heart transplants performed in India. Heart transplant involves a multi-disciplinary team effort with careful coordination between the different teams involved. This would also include meticulous monitoring in the post-operative period.

\section{PREOPERATIVE PREPARATION}

Almost all the recipients have severe impairment of Left ventricular function due to ischemic or other causes. In our institute of the eight recipients six had non ischemic dilated cardiomyopathy (DCM) ,followed by Ischemic DCM in one recipient and ischemic DCM with MR ( Mitral regurgitation ) in another recipient .Two of our cases were diabetic with optimal glycemic levels. As per ACC/ AHA(American college of cardiology / American Heart association) guidelines cardiac , respiratory evaluation, along with renal, liver, blood, coagulation parameters and serology are done. ${ }^{[1]}$ The Recipients are placed on Transplant waiting list, until appropriately sized and compatible ABO type donor organ is available.

\section{Gender distribution}

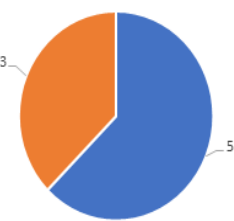

Indication for Heart transplant

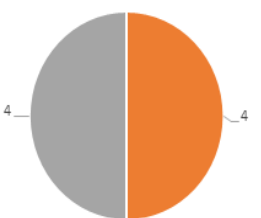

Optimizing patients waiting for transplant is mostly done on emergency basis. Whenever there is adequate time recipients are optimized maximally before surgery. Most of our cases had mildly elevated renal and liver function tests due to passive congestion. Anti-failure medications like diuretics, digoxin were titrated for response and continued throughout in all of them. Especially in patients with severe Right heart failure ,apart from history of alcoholism they were screened for ascites and evidence of cirrhosis. Presence of extensive liver fibrosis disqualifies them from cardiac transplant. ${ }^{[2,3,4]}$ In a study it was shown that combination of recipient age at time of heart transplant and Glomerular Filtration Rate value 1 year post-transplant can predict renal function post-transplant. ${ }^{[5,6]}$ One of our recipient had elevated pulmonary artery vascular pressure on hemodynamic assessment testing which was managed with use of pulmonary vasodilatorSildenafil. Pharmacological testing of reversibility of pulmonary hypertension is an essential test in evaluation and selection of candidates. Use of pulmonary vasodilator drugs perioperatively is crucial to reduce risk of early right heart failure. ${ }^{[7]}$ Some patients might require support systems as a bridge to transplant. ${ }^{[8,9,10,11]}$ The anaesthetic management of transplant cases is discussed along with the challenges encountered and outcomes in our centre.

Once the availability of donor is confirmed, the recipient is instructed to remain NPO. Irrespective of NPO status, rapid sequence induction was carried out in all patients. The donor heart is usually assessed by the harvesting team and the status is communicated to all the team members The operation theater is kept ready. A peripheral intra venous (iv) line is secured in the ward. when the harvesting team is convinced after examining the heart the recipient is shifted to operating room.

After shifting the recipient to operation theatre, monitors such as ECG, pulse oximetry and NIBP are attached.Antibiotic Inj. Meropenem $1 \mathrm{~g} \mathrm{IV}$ and methyl prednisolone 500mg IV are given before starting invasive lines. With strict aseptic precautions under local anesthesia radial artery cannulation is done with $20 \mathrm{G}$ catheter. 
Following this under local anesthesia using ultrasound guidance right IJV(Internal jugular vein) cannulation is done with a swan Ganz catheter for Pulmonary artery pressure monitoring .After measuring baseline PA Pressures, The PA catheter tip is placed only in right atrium and withdrawn further during explantation. After anastomoses of donor heart is complete it is advanced till right pulmonary artery. In Six cases we have used swan Ganz catheter and in 2 cases we have used vigileo cardiac output monitor. Vigilance calibration is done by providing Hemoglobin and $\mathrm{SvO} 2$ from gas analysis, results about indexed cardiac output, PVR are indexed to patient's weight. Other monitors used are BIS and cerebral oximetry and temperature probe in nasopharynx . Baseline hemodynamic parameters are noted ( HR , BP, MAP, PA pressure, Cardiac index and Stroke volume variation in case of using cardiac output monitor), then we proceed with induction.

Conduct of anesthesia - After preoxygenation patient is given Inj. midazolam $1 \mathrm{mg}$, fentanyl 4 micgm per $\mathrm{kg}$, etomidate $0.3 \mathrm{mg} / \mathrm{kg}$, rocuronium $1.5 \mathrm{mg} / \mathrm{kg}$ or vecuronium $0.1 \mathrm{mg} / \mathrm{kg}$. Patient is intubated with 7 or $8 \mathrm{~mm}$ oral cuffed endotracheal tube based on Patients requirement whether female or male. Of the cases one was Paediatric age 10 -year-old boy in whom all invasive lines were done after induction and intubation. In all cases, after confirming correct placement of tube and securing it , we proceed to cannulate femoral artery followed by femoral vein using triple lumen catheter . During induction fall in blood pressure is managed by bolus of phenylephrine (10 micrograms). In case of both fall in HR and blood pressure then ephedrine $3 \mathrm{mg}$ or $6 \mathrm{mg}$ bolus is given .Infusions of Dopamine, Nor adrenaline and Nitroglycerin are all kept ready. We maintained stable hemodynamics by use of (inotropes )supports and avoided extreme fluctuations.

\section{SURGICAL TECHNIQUE}

After sternotomy pericardium is opened. After heparinization ( 300 units $/ \mathrm{kg}$ ) ,Aorta, SVC and IVC cannulation done. Before SVC cannulation PA catheter is withdrawn from heart into the sterile sheath. Cardiopulmonary bypass started and core cooled. We use moderate hypothermia around 28 to $30 \mathrm{C}$. Hemofiltration and mannitol are used often as most patients have large blood volume and minimal renal impairment. Aorta is cross clamped. Explantation of native heart done. Donor heart transplant is done in orthotopic position using bicaval method. The advantage of this method is less incidence of rhythm disturbances and Tricuspid regurgitation. ${ }^{[12,13]}$ First Left atrial anastomoses is done followed by inferior vena cava, pulmonary artery, Superior vena cava then aorta in this order. After de airing, aortic cross clamp is released. Release of aortic cross clamp marks end of ischemic time of donor heart. Just before release of cross clamp, we administer $2^{\text {nd }}$ dose of methyl prednisolone $500 \mathrm{mg}$ IV to decrease chances of hyperacute rejection. ABG is reviewed and any electrolyte abnormalities or acidosis are corrected. Patient is rewarmed and Atrioventricular ( sequential) pacing done. The duration of surgery, total cardiopulmonary bypass time and aortic cross clamp times have been decreasing since we started three years ago. Initially for the first case the total duration of surgery was 7 hours with bypass time of 4 hours $42 \mathrm{mins}$ and cross clamp time of 2 hours $35 \mathrm{mins}$. The last case was done in 4 hours with a bypass time of 2 hours 50 mins and cross clamp time of 1 hour $45 \mathrm{mins}$.

\section{WEANING}

Since donor heart is denervated reflex response will be absent, Inotropes like nor adrenaline, adrenaline, dopamine along with nitroglycerin are started and titrated to response. Though most centres use isoprenaline, we were able to manage with these inotropes mostly dobutamine with adrenaline along with temporary epicardial atrial and ventricular pacing in all cases.

RV function can be usually assessed by direct observation (of the surgical field),(or) by) use of Trans esophageal echocardiography(TEE) . The other parameters which guided in smooth weaning were CVP, PA pressures and transpulmonary gradient. Inotropic support is done with adrenaline along with milrinone as well as inhaled nitric oxide and i.v prostaglandins. Transesophageal Echocardiography helped in overall assessment of the transplanted heart . (In ventilation) we maintain moderate hyperventilation and hyperoxia to prevent increase in pulmonary vascular resistance. We keep Fio2 around $60 \%$, tidal volume around 6 to $8 \mathrm{ml} / \mathrm{kg}$ with PEEP around 5.After adequate support time around 30 to 45 mins and when hemodynamic parameters are within normal limits and no evidence for right heart failure or rejection weaning is done. Decannulation and protaminisation done in routine way.

Goal directed loading of fluids were done based on hemodynamic and echo parameters. Crystalloids preferably ringer lactate is used . Albumin and fresh frozen plasma were used for maintenance of oncotic pressure. In all cases we have maintained hemoglobin around $10 \mathrm{~g} / \mathrm{dl}$ and above. Our target values are MAP less than $65 \mathrm{mmHg}$, CVP less than $12 \mathrm{mmHg}$, LAP around 8 to $12 \mathrm{mmHg}$, HR around 100 to 110 beats / $\mathrm{min}$, urine output more than $1.5 \mathrm{ml} / \mathrm{kg}$ and no acidosis in $\mathrm{ABG}$ with lactate less than $2 \mathrm{mmol} / \mathrm{L}$.

During closure wound infiltration is done with local anesthetic lignocaine along with bupivacaine in all cases. IV paracetamol $1 \mathrm{gm}$ along with $50 \mathrm{mg}$ tramadol is given for pain relief $6^{\text {th }}$ hourly in all cases. In the CT ICU, strict aseptic precautions are maintained to prevent infections as these patients are immunocompromised. Once they are stable hemodynamically and weaning criteria met, they are put on CPAP for 2 hours and if ABG is normal we planned for extubation The average duration for extubation post-transplant was $8-12 \mathrm{hrs}$.

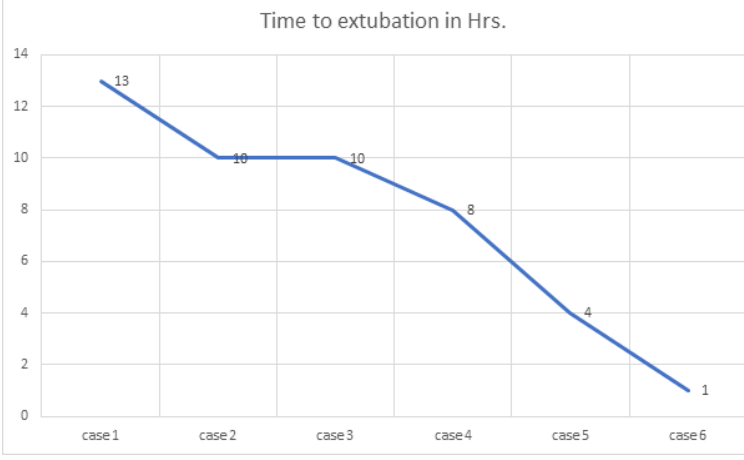

\section{DISCUSSION}

Of the cases only one recipient was in Paediatric age group. Paediatric heart transplant has evolved over the years to a well-established lifesaving procedure with excellent long-term outcome. It represents $14 \%$ of all cardiac transplants. In these cases, donor to recipient weight ratio must be considered. Echocardiogram gives very valuable information regarding anatomical and functional parameters of the donor heart. In general donor weight up to 2.5 times that of recipient are technically feasible. ${ }^{[14]}$ In our case the recipient was 10 -year-old boy weighing $30 \mathrm{~kg}$ and donor was a 25 -year-old male weighing $55 \mathrm{~kg}$. The donor heart was brought within a cold ischemic time of 15 mins . Graft survival and function are better when cold ischemic time is less than 180 mins. ${ }^{[15]}$ There are studies which have shown cold ischemic time is not a major concern in Paediatric heart transplants. ${ }^{[16]}$ The lines were all put after GA. The total bypass time was 2 hours $50 \mathrm{mins}$ and the cross-clamp time was 2 hours 5 mins. The surgical technique was also uneventful though some difficulty was anticipated in view of size disparity. Modification of technique may be needed in case of gross disparity in size. There was one episode of Generalized Tonic Clonic Seizure on $4^{\text {th }}$ POD for this child. It was managed with i.v phenytoin $100 \mathrm{mg}$. CT brain was also done, normal study. After opinion from neurologist we reduced the dose of cyclosporine .Rest of postoperative period was uneventful, he was discharged on $21^{\text {st }}$ POD and on regular follow up till date.

One of the patients developed acute kidney injury postoperatively, could not be normalized with fluids and diuretics so later went for hemodialysis on $3^{\text {rd }}$ POD . Post dialysis patient had arrest and could not be revived. Follow up of renal parameters and quick decision on RRT is crucial in these patients.

Another patient developed acute rejection and there was persistent low cardiac output not responding to high dose inotropes and increased immunosuppression. We lost that patient on $6^{\text {th }} \mathrm{POD}$.

In most of our cases donor selection was done with great caution almost all of them were below 50 years, majority were declared brain dead following road traffic accident. In the initial few transplants the warm ischemia time was a bit longer, once the surgical team is familiar with the technique, nowadays it is much shorter. For all cases follow up echo is done once immediate postoperatively then if no untoward events, repeated on $15^{\text {th }}$ POD by cardiologist and serum levels of 
cyclosporine are monitored in all cases.

\section{CONCLUSION}

To conclude careful selection of donor, adequate preload and good inotropic support during weaning, minimizing pulmonary vascular resistance and good pain relief are crucial for successful outcome. Rapid response is required in case of complications encountered postoperatively as well as attention to strict asepsis.

\section{REFERENCES}

1. Mariell Jessup, William T. Abraham, Donald E. Casey, Arthur M. Feldman, Gary S. Francis, Theodore G. Ganiats et al.2009 Focused Update: ACCF/AHA Guidelines for the Diagnosis and Management of Heart Failure in Adults. CIRCULATIONAHA. 109.192064 Circulation. 2009;119:1977-2016.

2. Ron-Bin Hsu, Chung-I Chang, Fang-Yue Lin, Nai-Kuan Chou, Nai-Hsin Chi, ShoeiShen Wang et al, Heart transplantation in patients with liver cirrhosis. European Journal of Cardio-Thoracic Surgery 2008;34(2):307-312.

3. Louie, C., Pham, M., Daugherty, T. et al. The liver in heart failure: a biopsy and explant series of the histopathologic and laboratory findings with a particular focus on precardiac transplant evaluation. Modern Pathology 2015;28: 932-943.

4. Dichtl,Vogel, W, unst, K.M.,Grander,W,Alber, H.F,Frick, M.et al, Cardiac hepatopathy before and after heart transplantation. Transplant International 2005;18: 697-702.

5. Björn Lindelöw, Claes-Håkan Bergh, Hans Herlitz, Finn Waagstein Jasn. Predictors and Evolution of Renal Function during 9 Years Following Heart Transplantation. Journal of American Society of Nephrology 2000;11(5): 951-957.

6. Hans Herlitz, Björn Lindelöw. Renal failure following cardiac transplantation. Nephrology Dialysis Transplantation,2000;15(3):311-314.

7. Klotz, Stefan \&Wenzelburger, Frauke\&Stypmann, Jörg\&Welp, Henryk \& Drees, Gabriele \& Schmid et al. Reversible Pulmonary Hypertension in Heart Transplant Candidates: To Transplant or Not to Transplant. The Annals of thoracic surgery.2006;82: 1770-3.

8. Donneyong M, Cheng A, Trivedi JR, et al. The association of pretransplant HeartMate II left ventricular assist device placement and heart transplantation mortality. ASAIO J 2014;60:294-299

9. Taghavi S, Jayarajan SN, Komaroff E, et al. Continuous flow left ventricular assist device technology has influenced wait times and affected donor allocation in cardiac transplantation. J Thorac Cardiovasc Surg 2014:147:1966-71

10. John R, Pagani FD, Naka Y, et al. Post-cardiac transplant survival after support with a continuous-flow left ventricular assist device: impact of duration of left ventricular assist device support and other variables. J Thorac Cardiovasc Surg 2010;140:174-81

11. Kamdar F, John R, Eckman P, et al. Postcardiac transplant survival in the current era in patients receiving continuous-flow left ventricular assist devices. J Thorac Cardiovasc Surg 2013;145:575-81

12. el Gamel A, Yonan NA, Grant S, Deiraniya AK, Rahman AN, Sarsam MA, et al. Orthotopic cardiac transplantation: a comparison of standard and bicaval Wythenshawe techniques. J Thora Cardiovascular Surg 1995;109(4):721 -9

13. Aziz T, Burgess M, Khafagy R, Wynn Hann A, Campbell C, Rahman A, et al. Bicaval and standard techniques in orthotopic heart transplantation: medium-term experience in cardiac performance and survival. J Thorac Cardiovasc Surg 1999;118(1):115-22.

14. Sethi GK, Lanauze P, Rosado LJ, Huston C, McCarthy MS, Butman S, et al. Clinical significance of weight difference between donor and recipient in heart transplantation. J Thorac Cardiovasc Surg 1993;106:444-8.

15. Zimmerman GJ, Johnston JK, Razzouk AJ, Gundry SR, Bailey LL.Duration of graft cold ischemia does not affect outcomes in pediatric heart transplant recipients. Scheule AM, Circulation. 2002;106(12):I163-7.

16. Kawauchi M(1), Gundry SR, de Begona JA, Fullerton DA, Razzouk AJ, BoucekM,Kanakriyeh M, et al. Prolonged preservation of human pediatric hearts for transplantation: correlation of ischemic time and subsequent function. J Heart Lung Transplant. 1993;12(1):55-8 